\title{
Role of Directional Wireless Communication in Vehicular Networks*
}

\author{
Ashish Agarwal and Thomas D.C. Little \\ Department of Electrical and Computer Engineering \\ Boston University, Boston, Massachusetts \\ \{ashisha, tdcl\}@bu.edu
}

May 17, 2010

MCL Technical Report No. 05-17-2010

\begin{abstract}
Enabling safety in vehicles is an ongoing challenge for the automotive sector. One approach towards enhancing safety is to increase knowledge within a vehicle of the actions of vehicles in the vicinity. Increased awareness is essential for activating the safety systems to take evasive or precautionary actions in the event of an incident. Wireless radio communication has emerged as a key enabler for exchanging safety information. Several initiatives across the world have considered various radio communication technologies to implement safety communication. However, there are significant constraints to utilizing wireless radio communication. In this article, we discuss briefly the challenges in enabling safety communication with wireless radio in the context of vehicular networks. We introduce the on-going work in utilizing free space optical communications as an enabler for inter-vehicle safety communication. As a first step, we compare with the current 802.11 standard implementation for achievable performance. Given that the two technologies are inherently different, directional versus omni-directional, we seek to identify the scenarios where each technology is best suited. Particularly, we compare packet delivery ratio (PDR), throughput and average packet delay of the two enabling technologies, under assumptions, in the context of increasing vehicle traffic density. Our results demonstrate that a directional technology such as free-space optics is less susceptible to contention scenarios. As a result, the performance in high density scenarios is better than that can be achieved from using omni-directional long-range technologies such as 802.11 .
\end{abstract}

${ }^{*}$ In Proc. IEEE Intelligent Vehicles Symposium (IV '10), San Diego, CA. June, 2010. This work is supported by the NSF under Grant Nos. CNS-0721884 and EEC-0812056. Any opinions, findings, and conclusions or recommendations expressed in this material are those of the author(s) and do not necessarily reflect the views of the National Science Foundation. 


\section{INTRODUCTION}

Next generation automotive safety technology could give us vehicles that are difficult to crash - and eventually may not need drivers at all [1]. Safety is an important application space for vehicular communication technology. As recently as Feb. 2010, Toyota vehicles are facing safety concerns [2].

While there has been advancement in vehicular safety technologies such anti-lock brake systems (ABS), traction control systems (TCS) and electronic stability control (ESC), that prevent accidents. These technologies can be further augmented by increasing awareness within a vehicle about actions of neighboring vehicles, by sharing state through wireless communication. Awareness beyond the line-of-sight can be efficiently enhanced by enabling vehicle-to-vehicle (V2V) and vehicle-to-infrastructure (V2I). It has been established in vehicular networking research, that applications can typically be characterized in 3 distinct classes; safety messaging, traffic and map-related applications and Internet access or general purpose data exchange. Each class of application has contrasting requirements for communication parameters of latency, bandwidth requirements and quality of service [3, 4].

Message dissemination in vehicular ad hoc networks (VANETs) is primarily enabled with wireless radio technologies. Several technologies such as cellular, 802.11 (Wi-Fi), Ultra-wide band (UWB) and free-space optics (FSO) have been explored [5]. We focus on comparing 802.11 and FSO techniques in this paper. Applications in VANETs such safety messaging, traffic and Internet access, [4, 6], have different requirements of bandwidth, latency and infrastructure. Internet access requires infrastructure such as access points or cellular towers for connectivity to the backbone network. Traffic applications have relatively relaxed latency constraints and involve collecting information from several sources (vehicles, road based sensors, highway cameras). Such applications can be instantiated without infrastructure support, enabled by multihop communication and networking. Safety communications, however, concern with exchanging state with nearest-neighbors to maintain safety in the system. As a result, the profile for data exchange is expected to be high frequency of updates with a small payload. A strict requirement is that of high reliability of messages delivered, that is, the packet delivery ratio (PDR) and the average delay of messages delivered. Thus, in the context of communication technologies, there will potentially be co-existing technologies for serving connectivity requirements in vehicular environments, much like Bluetooth is a connectivity technology while Wi-Fi is dedicated for Internet access.

The Dedicated Short Range Communication Spectrum (DSRC) has been allocated for development of 802.11p standard for inter-vehicle communication. The draft is a modification of the 802.11a standard that employs the use of DCF (Distributed Co-ordination Function). The implementation is a broadcast method to enable vehicles to share state information in a fast and efficient manner with minimal setup time. The broadcast methodology, creates contention for the wireless medium in scenarios where the vehicular traffic density is high. Coupled with the potentially high frequency of data exchange, this creates a 'Broadcast Storm' scenario, where there are several nodes broadcasting at high frequency, effectively jamming the wireless space [7]. This is illustrated in Figure 1 where an incident on a dense highway energizes safety communication between vehicles, thereby, creating a broadcast storm. Contention in these scenarios causes increased number of packets dropped and increasing data delivery delays. For safety-critical communication, this is non-ideal for the reliability goals of the system [8].

There is related work in developing high frequency radio techniques that are directional [9]. The advantages of this technology are that it limits the contention and provides directionality and context in communication. Our group is currently developing transceivers based on Free Space Optical (FSO) communication that enable directional, line-of-sight communication. In the context of safety applications, we explore the performance FSO for packet delivery, average delay and throughput especially in the context of dense vehicular density scenarios. We use modeling techniques developed in references [10, 11], to develop a model for performance of 802.11 in broadcast mode and compare with a vision of a similar technique implemented by directional communication such that the contention problems are prevented by using directional commu- 


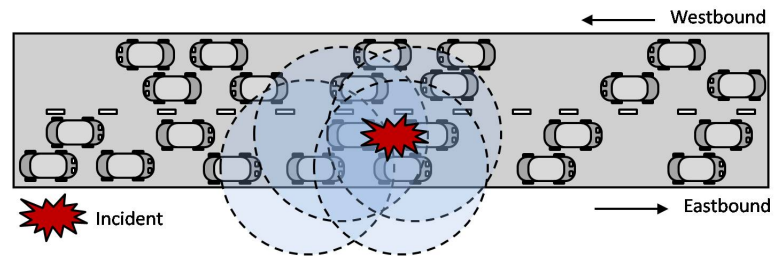

Figure 1: Figure illustrating the 'Broadcast Storm' problem. An incident occurring on a dense highway energizes high priority messaging between vehicles leading to contention in the network.

nication.

Our goal for this work is to evaluate the feasibility of using free-space optical (FSO) communication for the use of safety messaging in high density scenarios. The rest of this paper is organised as follows: Section 2 describes the related work in the context of analytical modeling of safety message propagation and freespace optical communication. Section 3 describes the assumptions of our model for evaluation. We present the simulation results in Section 5 and conclude the paper with a discussion in Section 6.

\section{RELATED WORK}

The DARPA Grand Challenge, [12] is an autonomous driving vehicle challenge that seeks to develop technologies that can enable actuator systems, enabled by sensors, to drive autonomously. Google engineers have a similar vision for the future of driving [13]. However, these systems are heavily dependent upon self-sensors for implementation and don't exploit the potential for inter-vehicle data exchange. Our proposed system seeks to enable a communication framework that will enable higher resolution of awareness in the system that will potentially lead to autonomous driving vehicles through coordination between vehicles. Researchers at USP [14] are working on developing mathematical models for autonomous driving and have expressed the importance on the role of communication in future vehicles.

Directional communication technologies are considered to enhance or mitigate problems experienced in omni-directional technologies. Examples include 60GHz radio, [9], Free Space Optics (FSO), and Steering antennas of 802.11 radios to optimize performance [15]. In reference [15], authors develop a framework to use antenna steering to optimize the performance of the radio when connecting to a roadside access point (AP). The authors demonstrate that significant gains can be achieved in the throughput by improving connectivity duration and the signal to noise ratio (SNR). Several works have considered the use of free-space optical communication in the context of vehicular networking. The Visual Light Communication Consortium (VLCC) in Japan is developing an LED traffic light based data dissemination system that modulates the LEDs at high rates to disseminate data to vehicles that have receivers in the form of high speed cameras [16]. Intel has demonstrated an active-braking application using LED communication between vehicles [17]. Our group is currently working on developing optical transceivers that can be potentially installed on vehicles an enable directional, line-of-sight vehicle-to-vehicle communication.

DSRC or Dedicated Short Range Communication Spectrum is a $5.9 \mathrm{GHz}$ frequency allocation for developing WAVE (Wireless Access in Vehicular Environments) [18]. The 802.11p is a draft being developed to standards and protocols for communication between vehicles and road-side infrastructure such as access points [8]. In the context of 802.11 modeling, there have been several works that model the real-world observation very closely. In particular we refer to the work by Bianchi [10] in developing a Markov model for the 802.11 DCF (Distributed co-ordination function). Authors in reference [11, 19, 20] have modeled the steady-state performance of 802.11 under saturated and unsaturated conditions. Authors in [7], have extensively studied the broadcast storm problem in an implementation using 802.11 and suggested modifications 
to the draft. Another technique proposed in [21], modulates the transmission power of the radio in dense scenarios to avoid contention due to large number of nodes in the system. We adapt the models developed in these works and extend these assumptions to the free-space optical communication work. We compare the performance of free-space optical communication under the assumption of similar performance parameters. Part of our goal is to develop a system that can match these parameters if not exceed in performance given the physical constraints of line-of-sight. Through this work we understand the strengths and weakness of the two technologies under consideration.

\section{Vehicular Network Scenario}

We illustrate a model of our system in Figure 2. We assume a simple bi-directional linear model of the highway, where vehicles travel in opposing directions. Vehicles are equipped with optical transceivers such that they can communicate with other similarly equipped vehicles. The difference from wireless radio technologies being, the communication is directed and limited by line-of-sight.

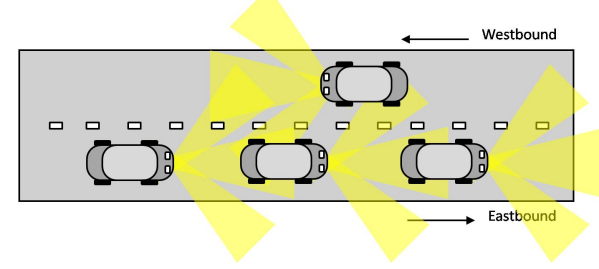

Figure 2: Figure illustrating directional communication implemented with free-space optical communication.
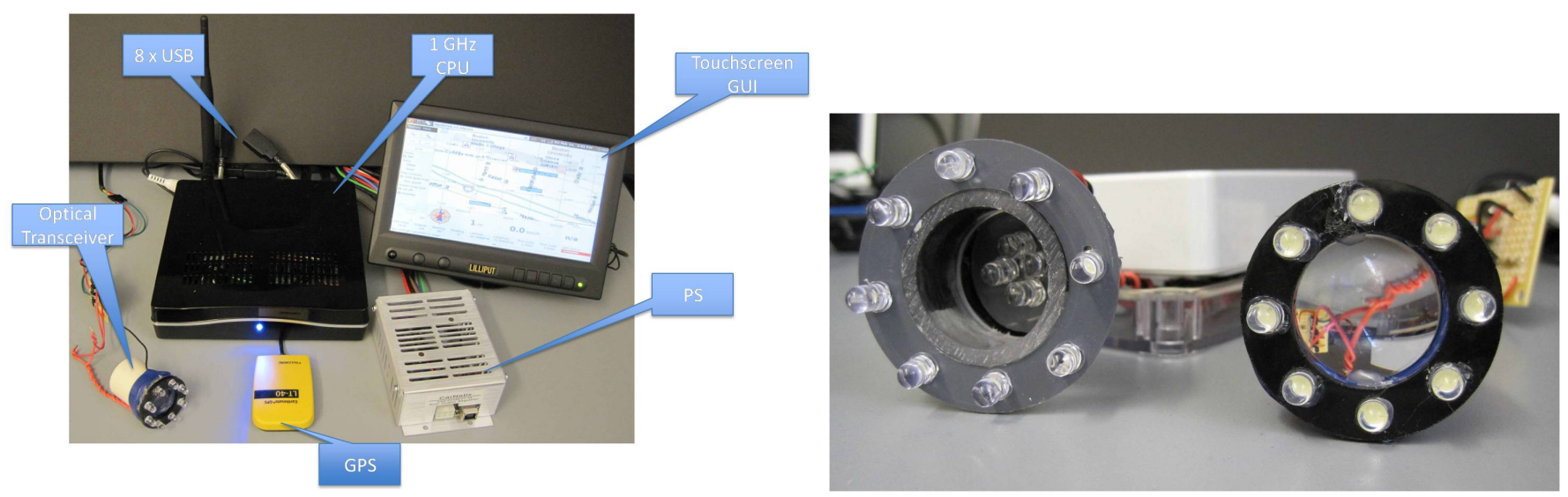

Figure 3: Pictures from our current system under development - (a) A car computer that is connected to the optical transceivers to demonstrate information exchange (b) Detailed view of the optical transceiver design

In Figure 3(a), we show a car computer integrated with our optical transceivers. The car computer is an off-the-shelf component that is equipped with a touch-enabled screen for visual display, a fan-less CPU for operation in harsh environments, and wireless communication capability [22]. The device has eight USB ports for connectivity to various devices. Our optical transceivers are connected to this device to demonstrate information exchanged with other vehicles in the system.

In Figure 3(b), we show a detailed picture of our optical transceivers. The transceiver design has eight white LEDs as transmitters, 4 each in parallel that are modulated at high frequency for signaling. The 
modulation rate is high such that it is not perceivable by the human eye. Currently, the system is able to achieve a data rate of $1 \mathrm{Mbps}$, under assumptions. As a receiver we have photo-diodes at the center equipped with a lens to minimize visible light interference from the transmitter itself and concentrate the received signal at the photo-diodes. The targeted range is $50 \mathrm{~m}$. Since the communication is directed and restricted by line-of-sight (nearest neighbor communication), the contention at each individual receiver is limited.

In the context of mathematical modeling, we rely on prior works that have been shown to be in strong agreement with real-world experiments $[10,11]$. We extend these models to study the vehicular networking scenario with high density bi-directional traffic and high packet rates. We are primarily concerned with contention and focus on the analytical results based on the models. We assume that nodes are exponentially distributed on the roadway, consistent with related work [23]. We concentrate our analysis on density distribution of vehicles, ranging from a sparse density of 10 vehicles $/ \mathrm{km}$ to a dense scenario of 100 vehicles $/ \mathrm{km}$. We assume a broadcast scenario where vehicles exchange state information. Given the high mobility rates of vehicles in the system and the relatively random arrivals and departures of nodes in the system, we seek to minimize the connection establishment time by assuming a broadcast mechanism. We assume the system is in saturation mode because vehicles are constantly exchanging state information. The saturation mode is described as the state of the system such that, each node in the network has a packet immediately available for transmission after the completion of each transmission. Thus, the queue at each node is not empty and each packet has a random backoff time before it is successfully transmitted. As a result, there is high frequency of data exchange, thus, even with a small number of nodes in the system, there is a state of saturation. This assumption is distinct from related work that do not always assume a high data rate. We use the saturation mode, as we envision a system of nodes that is continuously interacting with other nodes within transmission range, potentially sharing state information for the purpose of maintaining safety through situational awareness.

\section{Analytical Model}

We briefly describe the models used in related work [11] to derive the quantities of interest. Particularly, we want to study the average delay and packet delivery ratios for developing a communication sub-system that can satisfy the reliability constraints of safety-critical communication.

\section{Packet Delivery Ratio}

The packet delivery ratio (PDR) is defined as the ratio of number of packets successfully received to the number of packets transmitted. Using the model given in [11]:

$$
P D R=\frac{n \tau(1-\tau)^{n-1}}{n \tau}=\left(\frac{W-1}{W+1}\right)^{n-1},
$$

where $n$ is the number of nodes in the range of a vehicle, $\tau$ is the probability a station transmits in the time slot, $W$ is the backoff window size of backoff counter for DCF. The parameter $\tau$ is given by:

$$
\tau=\frac{2}{W+1}
$$

\section{Packet Delay}

Saturation packet delay is the average delay that a packet experiences between the time at which the packet is generated and the time at which the packet is successfully received under saturation conditions. It includes 
the medium access delay (due to backoff, channel busy, inter-frame spaces, etc.), transmission delay and propagation delay. The average delay for a successfully transmitted packet $E[D]$ is given by:

$$
E[D]=\frac{W+1}{2}\left[\left(1-p_{b}\right) \sigma+p_{s} T_{s}+p_{c} T_{c}\right]
$$

where $T_{s}$ is the average time that a channel is sensed busy due to a successful transmission and $T_{c}$ is the average time a channel is sensed busy due to a collision. The quantities are derived as:

$$
T_{s}=T_{c}=(M A C+P H Y+P) / R+D I F S+\delta
$$

where $M A C, P H Y$ denote the MAC and PHY Header size, DIFS is the Distributed Inter-Frame Space. $\delta$ is the propagation delay and $R$ is the channel bit-rate.

$p_{b}$ is the probability that the channel is busy, given by:

$$
p_{b}=1-(1-\tau)^{n} \text {. }
$$

$p_{s}$ is the probability that a successful transmission occurs in a time slot derived as:

$$
p_{s}=n \tau(1-\tau)^{n-1}
$$

Finally, $p_{c}=p_{b}-p_{s}$.

\section{Normalized Throughput}

The normalized saturation throughput $(\mathrm{S})$ is defined as the fraction of time at which the channel is used to successfully transmit payload bits.

$$
S=\frac{p_{s} P}{\left(1-p_{b}\right) \sigma+p_{s} T_{s}+p_{c} T_{c}}
$$

\section{Simulation Results}

Our goal for this work is to analytically determine the trade-offs between utilizing wireless 802.11 versus using free-space optical communication (FSO) for vehicular communication applications. We use the model for 802.11 broadcast mechanism in the saturation state given in [11], for computing the packet delivery ratio, packet delay and throughput for increasing node density in the network and compare assuming similar parameters for FSO. The 802.11 radio standard, typically, has a range of $200 \mathrm{~m}$, while the FSO is expected to have a communication range of $50 \mathrm{~m}$. The assumption is that the wireless radio is omni-directional while the FSO is a directional communication. The two technologies are inherently different, we seek to compare the behavior under assumptions and the influence of parameters on the performance. The traffic density is varied from a low of 10 vehicle $/ \mathrm{km}$ to a high of 100 vehicles $/ \mathrm{km}$. An exponential distribution of nodes on a liner roadway is assumed, consistent with prior work [23]. The simulation parameters for the 802.11 and FSO modeling are given in Table 1

In Figure 4(a), we show a comparison of the number of nodes in the neighborhood of a vehicle that is equipped with 802.11 or FSO technology. Considering a bi-directional highway scenario with a similar traffic density, there are potentially 80 vehicles in a neighborhood. However, when considering a short-range technology, the range is $50 \mathrm{~m}$ limits reception of data from vehicles that are beyond a vehicle's immediate neighborhood. Thus, at the extreme density of 100 vehicles $/ \mathrm{km}$, the number of nodes in a neighborhood of a single receiver are 10, on average. Thus, it is trivial to note, there is an advantage to using directional wireless techniques, especially in high density scenarios. 
Table 1: Parameters used in 802.11 modeling

\begin{tabular}{|l|l|}
\hline Parameter & Value \\
\hline Packet Payload, $P$ & 8184 bits \\
\hline MAC Header, $M A C$ & 272 bits \\
\hline PHY Header, $P H Y$ & 128 bits \\
\hline Channel bit rate, $R$ & $1 \mathrm{Mbit} / \mathrm{s}$ \\
\hline Propagation Delay, $\delta$ & $1 \mu s$ \\
\hline DIFS & $128 \mu s$ \\
\hline Slot time $\tau$ & $50 \mu s$ \\
\hline Backoff window size, $W$ & $32,64,128$ \\
\hline
\end{tabular}
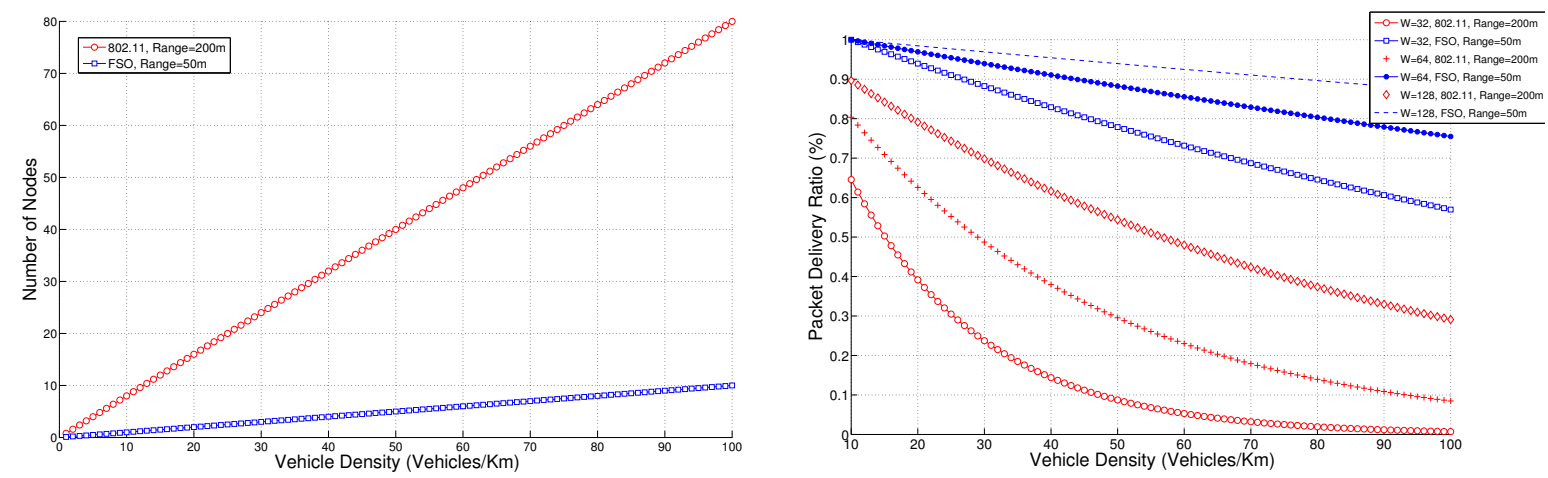

Figure 4: Analytical Results - Comparison of wireless 802.11 and FSO techniques (a) Number of nodes in a neighborhood (b) Packet Delivery Ratio.

In Figure 4(b), we show the comparison of packet delivery ratios (PDR) as the number of nodes in the network increase. For the 802.11, the PDR rapidly decreases as there is increasing contention for the wireless medium, while the FSO technique has potentially limited contention for the limited range and property of directionality. As the backoff window $(W)$ is increased, the packet delivery ratio is higher. However, even with increasing back-off window size $W$, the packet delivery ratio falls below $50 \%$. For the FSO implementation, the packet delivery rate is relatively higher and only falls below $80 \%$ in very high density scenarios.

In Figure 5(a), we show the average packet delay in data exchange. For the 802.11 technique, packet delay is at a minimum 0.05 seconds in low density scenarios and up to a high of 0.3 seconds in high density scenarios. The packet delay increases because as the contention in the medium increases, nodes have to wait for a long time due to collisions and backoff. Increasing the backoff window size increases the delay as the nodes have to wait for more time slots. For FSO, the delay is always below 0.05 seconds. It has been established in related work that packet delay in vehicular communication for safety applications should be strictly below $400 \mathrm{~ms}$ [8]. While the average here meets the criteria, there are scenarios where this criteria is not achievable. Thus, backoff window size $(W)$ is an important design consideration in protocols for safety communication.

In Figure 5(b), we show the normalized throughput. The results show a similar trend to the packet 

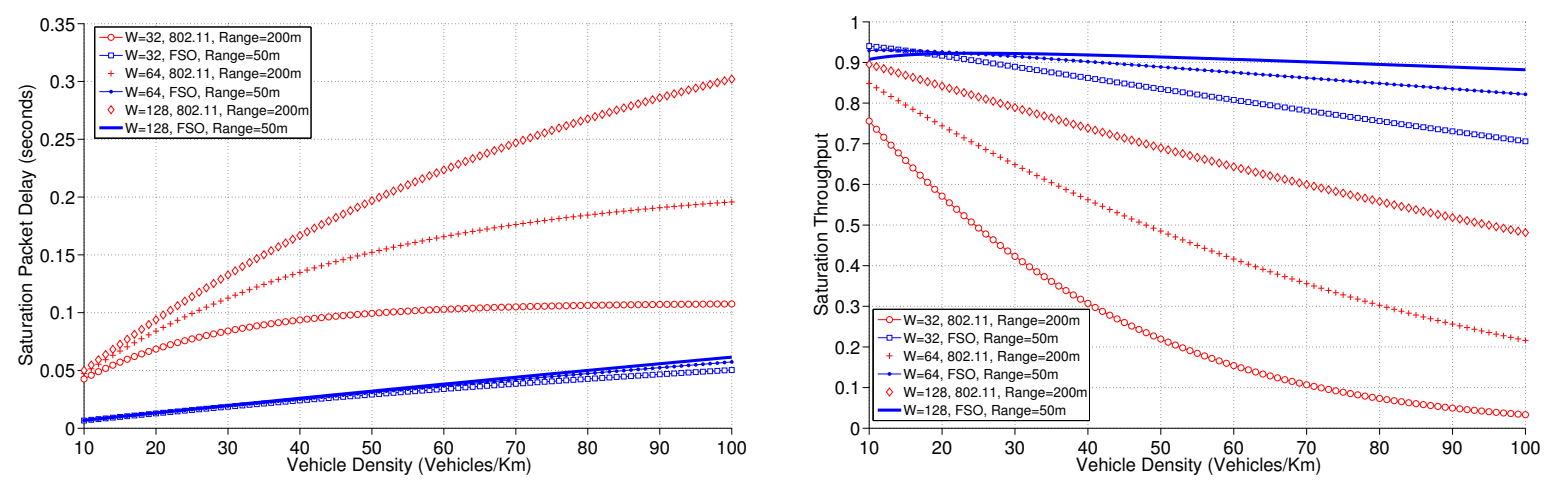

Figure 5: Simulation Results for 802.11 (range=200 m) and FSO (range=50 m) - (a) Delay per packet in seconds (b) Normalized Throughput.

delivery ratio (PDR) for 802.11. It is trivial to note that the as the collisions increase, the packet delivery ratio will decrease and the channel utilization is low. By increasing, the backoff window size $(W)$, the packet collisions can be prevented, thereby increasing channel utilization. It is interesting to note for the FSO, for $W=32$, the channel utilization rate actually increases. This is due to the observation that the nodes are waiting in backoff mode while the channel as the number of nodes competing for the channel are low. The drop in throughput is not as severe as that in 802.11.
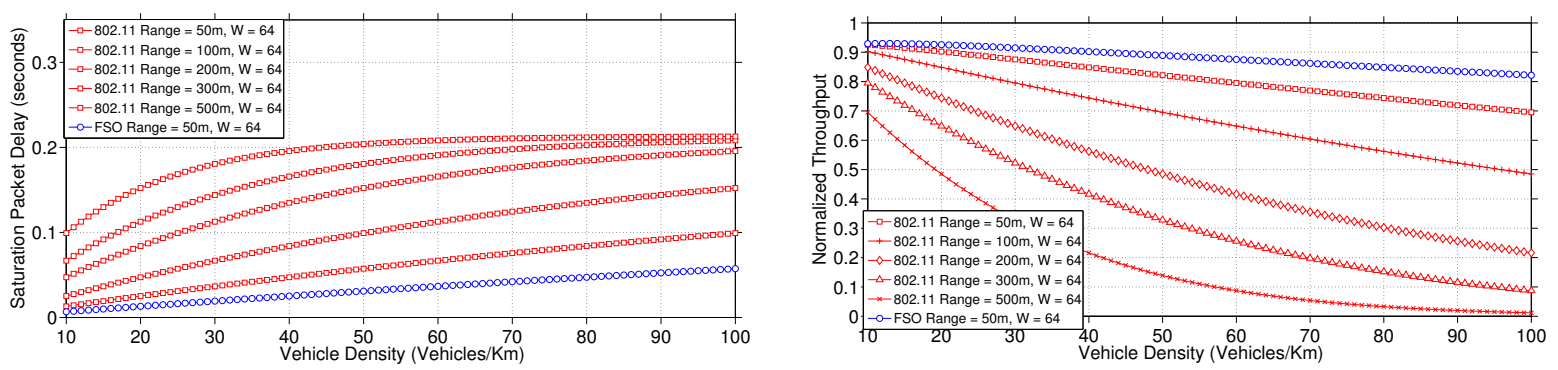

Figure 6: Simulation Results for 802.11 (variable range) and FSO (range=50 m) - (a) Delay per packet in seconds (b) Normalized Throughput.

In Figure 6(a), we compare the packet delays for different radio range values for 802.11 . By controlling the transmission power of the radio, a larger or smaller transmission range can be achieved. Correspondingly, the contention increases or decreases. However, due to the omni-directional properties of the radio, the number of nodes and the corresponding contention is higher than a directional technology like FSO. A similar trend is observed in the Throughput performance (Fig. 6(b)). The throughput decreases rapidly with increasing radio range.

\section{Discussion AND CONCLUSION}

In this work, we have shown analytically the potential trade-offs between utilizing 802.11 radios versus using short-range, directional communication implemented by free-space optics. Wireless 802.11 standard has potentially higher range of $200 \mathrm{~m}$, as defined for vehicular communication. While the free-space optical communication is expected to offer $50 \mathrm{~m}$ communication range. Applications such as safety and emergency messaging require very high reliability. However, due to the shared medium access and application of 
broadcast mechanisms for wireless communication, the probability of a successful transmission decreases exponentially with increasing node density in the network. This phenomenon is characterized as the broadcast storm problem in ad hoc networks. This is true for both the wireless 802.11 and FSO technologies. However, FSO being a short-range, has inherent benefits of directional technology, the interference from high density of nodes in the network is less than that experienced by the omni-directional wireless 802.11. As observed from the results, the average packet delay is approximately 0.3 seconds for high density scenarios which is unsuitable for data delivery constraints in design of safety applications in vehicular networks. One strategy is to use a short-range directional communication technique that limits contention and offers benefits of directionality. Our preliminary analysis provides an overview of the performance criteria that can be achieved using directional techniques. Further, we study the parameters such as backoff window size $(W)$ that determine the packet delivery ratio, delay and throughput achieved. It is interesting to note the trade-off between delivery ratio and delay. By increasing the backoff window, collisions can be avoided, however, the trade-off is an increased delay in packet delivery. Thus, it is an important design consideration for any safety protocol for vehicular communication. The requirement is of a communication subsystem that guarantees packet delivery within delay limits.

\section{References}

[1] S. Ashley, "Crashless cars: Making driving safer," in Scientific American Magazine, December 2008.

[2] N. Bunkley. (2010, March) U.S. Starts Inquiry Into Brake Problems on Prius. [Online]. Available: http://www.nytimes.com/2010/02/05/business/global/05toyota.html

[3] A. Agarwal and T. D. C. Little, "Prospects for Networked Vehicles of the Future," in Proc. Smart Transportation Workshop in IEEE Real-Time and Embedded Technology and Applications Symposium (RTAS), Bellevue, WA, USA, April 2007.

[4] T. D. C. Little and A. Agarwal, "Connecting Vehicles to 'The Grid'," in Proc. NITRD National Workshop on High-Confidence Automotive Cyber-Physical Systems, Troy, MI, April 2008.

[5] Y. Zang, S. Sories, G. Gehlen, and B. Walke, "Towards a European Solution for Networked Cars - Integration of Car-to-Car technology into cellular systems for vehicular communication in Europe," Speech, ITU, Geneva, Switzerland, p. 14, Mar 2009. [Online]. Available: http://www.comnets.rwth-aachen.de

[6] T. D. C. Little and A. Agarwal, "An Information Propagation Scheme for Vehicular Networks," in Proc. IEEE Intelligent Transportation Systems Conference (ITSC), Vienna, Austria, September 2005, pp. $155-160$.

[7] O. Tonguz, N. Wisitpongphan, J. S. Parikh, F. Bai, P. Mudalige, and V. Sadekar, "On the Broadcast Storm Problem in Ad hoc Wireless Networks," oct. 2006, pp. $1-11$.

[8] ASTM E2213-03, "Standard Specification for Telecommunications and Information Exchange Between Roadside and Vehicle Systems - $5 \mathrm{GHz}$ Band Dedicated Short Range Communications (DSRC) Medium Access Control (MAC) and Physical Layer (PHY) Specifications," ASTM Std., Tech. Rep., September 2003.

[9] R. Daniels and R. Heath, "60 GHz Wireless Communications: Emerging Requirements and Design Recommendations," IEEE Vehicular Technology Magazine, vol. 2, no. 3, pp. 41-50, Sept. 2007. 
[10] G. Bianchi, "Performance Analysis of the IEEE 802.11 Distributed Coordination Function," IEEE Journal on Selected Areas in Communications, vol. 18, no. 3, pp. 535-547, Mar 2000.

[11] X. Ma and X. Chen, "Performance Analysis of IEEE 802.11 Broadcast Performance Analysis of IEEE 802.11 Broadcast Scheme in Ad Hoc Wireless LANs," in IEEE Transactions On Vehicular Technology, vol. 57, no. 6, November 2008.

[12] L. Grossman. (2008, March) Building the Best Driverless Robot Car. [Online]. Available: http://www.time.com/time/magazine/article/0,9171,1684543,00.html

[13] J. Ramsey. (2009, July) Google phone designers plot for "the end of driving" with autonomobile. [Online]. Available: http://www.autoblog.com/2009/07/08/ google-phone-designers-plot-for-the-end-of-driving-with-autono/

[14] USP researchers say future cars will communicate to avoid collisions. [Online]. Available: http://www.usp.ac.fj/news/story.php?id=416

[15] V. Navda, A. P. Subramanian, K. Dhanasekaran, A. Timm-Giel, and S. Das, "MobiSteer: Using Steerable Beam Directional Antenna for Vehicular Network Access," in Proceedings of the 5th International Conference on Mobile Systems, Applications and Services (MobiSys '07). New York, NY, USA: ACM, 2007, pp. 192-205.

[16] S. Arai, S. Mase, T. Yamazato, T. Endo, T. Fujii, M. Tanimoto, K. Kidono, Y. Kimura, and Y. Ninomiya, "Experimental on Hierarchical Transmission Scheme for Visible Light Communication using LED Traffic Light and High-Speed Camera," in VTC Fall, 2007, pp. 2174-2178.

[17] K. Greene. (2009, June) A vision for headlight communications. [Online]. Available: http: //technologyreview.com/

[18] Q. Xu, T. Mak, R. Sengupta, and J. Ko, "Vehicle-to-Vehicle Safety Messaging in DSRC," in Proc.1st ACM Workshop on Vehicular ad hoc networks, Philadelphia, USA, October 2004, pp. 19-28.

[19] J. An, X. Guo, and Y. Yang, "Analysis of collision probability in vehicular ad hoc networks," in GEC '09: Proceedings of the first ACM/SIGEVO Summit on Genetic and Evolutionary Computation. New York, NY, USA: ACM, 2009, pp. 791-794.

[20] V. Gau, C.-W. Huang, and J.-N. Hwang, "Reliable Multimedia Broadcasting over Dense Wireless Ad Hoc Networks," in invited survey paper for Special Issue on Multimedia Communications, Networking, and Applications, . Journal of Communications (JCM), Academic Publisher, October 2009, pp. 614627.

[21] J. Mittag, F. Schmidt-Eisenlohr, M. Killat, J. Härri, and H. Hartenstein, "Analysis and Design of Effective and Low-overhead Transmission Power Control for VANETs," in Proceedings of the fifth ACM International Workshop on VehiculAr Inter-NETworking (VANET '08). New York, NY, USA: ACM, 2008, pp. 39-48.

[22] (2010, March) Micro Intel Vehicle \& Home Media Computer. [Online]. Available: $\{\{$ http: //store.mp3car.com/Ultimate $\backslash$ Micro $\backslash$ Intel $\backslash \_$Vehicle $\backslash \_$Computer $\backslash$ pp/ccc-208.htm $\left.\}\right\}$

[23] R. Fracchia and M. Meo, "Analysis and Design of Warning Delivery Service in Intervehicular Networks," IEEE Transactions on Mobile Computing, vol. 7, no. 7, pp. 832-845, 2008. 
[24] A. Agarwal, D. Starobinski, and T. D. C. Little, "Analytical Model for Message Propagation in Delay Tolerant Vehicular Ad Hoc Networks," in Vehicular Technology Conference (VTC-Spring '08), Singapore, May 2008, pp. 3067-3071.

[25] X. Ma, X. Chen, and H. H. Refai, "Performance and reliability of DSRC vehicular safety communication: a formal analysis," EURASIP Journal on Wireless Communication Networks, vol. 2009, pp. $1-13,2009$. 